PROCEEDINGS OF THE

AMERICAN MATHEMATICAL SOCIETY

Volume 129, Number 8 , Pages 2309-2318

S 0002-9939(01)05839-7

Article electronically published on January 17, 2001

\title{
CRITERIA OF PÓLYA TYPE FOR RADIAL POSITIVE DEFINITE FUNCTIONS
}

\author{
TILMANN GNEITING
}

(Communicated by Christopher D. Sogge)

\begin{abstract}
This article presents sufficient conditions for the positive definiteness of radial functions $f(x)=\varphi(\|x\|), x \in \mathbb{R}^{n}$, in terms of the derivatives of $\varphi$. The criterion extends and unifies the previous analogues of Pólya's theorem and applies to arbitrarily smooth functions. In particular, it provides upper bounds on the Kuttner-Golubov function $k_{n}(\lambda)$ which gives the minimal value of $\kappa$ such that the truncated power function $\left(1-\|x\|^{\lambda}\right)_{+}^{\kappa}, x \in \mathbb{R}^{n}$, is positive definite. Analogous problems and criteria of Pólya type for $\|\cdot\|_{\alpha}$-dependent functions, $\alpha>0$, are also considered.
\end{abstract}

\section{INTRODUCTION}

The real-valued function $f$ defined on the $n$-dimensional Euclidean space $\mathbb{R}^{n}$ is positive definite if the matrix

$$
\left(f\left(x_{i}-x_{j}\right)\right)_{i, j=1}^{k}
$$

is nonnegative definite for all finite systems $x_{1}, \ldots, x_{k}$ of points in $\mathbb{R}^{n}$. It is radial or isotropic if $f(x)=\varphi(\|x\|), x \in \mathbb{R}^{n}$, for some function $\varphi:[0, \infty) \rightarrow \mathbb{R}$ and $\|\cdot\|$ the Euclidean norm on $\mathbb{R}^{n}$. Radial positive definite functions have significant applications in probability theory, statistics, and approximation theory, where they occur as the characteristic functions or Fourier transforms of spherically symmetric probability measures, as the covariance functions of stationary and isotropic random fields, and as the radial basis functions in scattered data interpolation.

Let $\Phi_{n}, n=1,2, \ldots$, denote the class of the continuous functions $\varphi:[0, \infty) \rightarrow \mathbb{R}$ which are such that $\varphi(0)=1$ and the radial function $f(x)=\varphi(\|x\|), x \in \mathbb{R}^{n}$, is positive definite. Thus, we may identify $\Phi_{n}$ with the class of the characteristic functions of $n$-variate, spherically symmetric probability distributions, or with the class of the continuous correlation functions of stationary and isotropic random fields on $\mathbb{R}^{n}$. In a classical paper, Schoenberg [27] showed that $\varphi$ is an element of the class $\Phi_{n}$ if and only if it is of the form

$$
\varphi(t)=\Gamma(n / 2) \int_{[0, \infty)}\left(\frac{2}{r t}\right)^{(n-2) / 2} J_{(n-2) / 2}(r t) d F(r) \quad(t \geq 0)
$$

Received by the editors November 29, 1999.

2000 Mathematics Subject Classification. Primary 42B10, 60E10, 42A82.

(C)2001 American Mathematical Society 
for $F$ a probability measure on $[0, \infty)$ and $J_{(n-2) / 2}$ a Bessel function of order $(n-2) / 2$. If the radial function associated with $\varphi$ is integrable over $\mathbb{R}^{n}$, then $\varphi$ admits the representation (1) if and only if the Bessel integral

$$
\widehat{\varphi}(s)=\left(\frac{\pi s}{2}\right)^{1 / 2} \int_{0}^{\infty} \varphi(t) t^{n / 2} J_{(n-2) / 2}(t s) d t
$$

is nonnegative for $s>0$ (see Bochner and Chandrasekharan 4, Section II.7). Yet very often it is impossible to check directly whether $\widehat{\varphi}(s)$ is nonnegative. Thus, simple sufficient conditions for the positive definiteness of radial functions have been sought. The prototype for a result of this kind is the celebrated criterion of Pólya ([24, see also Sasvári [26]): if $\varphi:[0, \infty) \rightarrow \mathbb{R}$ is a continuous and convex function with $\varphi(0)=1$ and $\lim _{t \rightarrow \infty} \varphi(t)=0$, then $\varphi$ belongs to the class $\Phi_{1}$. Pólya's criterion is beautiful and has been widely applied. Nevertheless, it applies only in the univariate case and only to functions $f(x)=\varphi(|x|), x \in \mathbb{R}$, which are not differentiable at zero. The key result of this paper is the following criterion. It comprises all dimensions and applies to arbitrarily smooth radial functions.

Theorem 1.1. Let $\varphi:[0, \infty) \rightarrow \mathbb{R}$ be a continuous function with $\varphi(0)=1$ and $\lim _{t \rightarrow \infty} \varphi(t)=0$. Suppose that $k$ and $l$ are nonnegative integers, at least one of which is strictly positive. Put

$$
\eta_{1}(t)=\left.\left(-\frac{d}{d u}\right)^{k} \varphi(\sqrt{u})\right|_{u=t^{2}} .
$$

If there exists an $\alpha \geq 1 / 2$ so that

$$
\eta_{2}(t)=\left(-\frac{d}{d t}\right)^{k+l-1}\left[-\eta_{1}^{\prime}\left(t^{\alpha}\right)\right]
$$

is convex for $t>0$, then $\varphi$ is an element of the class $\Phi_{n}$, for $n=1, \ldots, 2 l+1$.

The theorem extends and unifies the previous analogues of Pólya's criterion for radial functions. Specifically, the following cases have been known.

$\mathrm{k}=\mathbf{0}, \mathbf{l} \geq \mathbf{1}, \alpha=1$ : This beautiful and widely known result of Askey [1] (see also Letac and Rahman [15] and Trigub [29]) covers all dimensions.

$\mathrm{k}=\mathbf{0}, \mathrm{l} \geq \mathbf{1}, \alpha=1 / 2$ : This criterion is stronger than the corresponding result for $\alpha=1$, but still applies to convex functions $\varphi$ only. It was established by Mittal [22] and Gneiting [7] in seemingly different albeit equivalent forms.

$\mathrm{k}=1, \mathrm{l}=\mathbf{0}, \alpha=\mathbf{1} \mathbf{2}$ : This univariate result of Gneiting [9] is the only previously known analogue of Pólya's theorem that applies to functions which are differentiable at zero.

Note that Theorem 1.1 puts a condition on a derivative of order $2 k+l$. In particular, it assumes that $\varphi^{(2 k+l)}(t)$ exists for $t>0$. This type of result is the best we can possibly hope for. We will see later on that the criterion applies to radial functions $f(x)=\varphi(\|x\|)$ in $\mathbb{R}^{2 l+1}$ which are differentiable of order $2 k$ at the origin. Gneiting [6] shows that if $f$ is positive definite, then $\varphi^{(2 k+l)}(t)$ exists for $t>0$. The convexity condition on $\eta_{2}$ can be checked very efficiently, by taking derivatives two more times and checking for nonnegativity. Using computer algebra systems, this lends itself to simple and powerful tests for positive definiteness. Finally, the criterion can also be used to check whether a radial function has a unimodal Fourier transform (compare Askey [2] and Section 4 of Gneiting [5]). 
The remainder of the article is organized as follows. Section 2 supplies further comments as well as the proof of the criterion. The idea for the proof is straightforward and based on the well-known fact that scale mixtures of positive definite functions are positive definite. In Section 3] we apply the criterion to Kuttner's [14] and Golubov's [10] question whether the truncated power function $\varphi(t)=\left(1-t^{\lambda}\right)_{+}^{\kappa}$ is an element of $\Phi_{n}$. Section 4 concludes the paper and concerns the analogous problem in the more general case of positive definite functions $f(x)=\varphi\left(\|x\|_{\alpha}\right)$, $x \in \mathbb{R}^{n}$, which depend on an $l_{\alpha}$-(quasi)norm

$$
\|x\|_{\alpha}=\left(\left|x_{1}\right|^{\alpha}+\cdots+\left|x_{n}\right|^{\alpha}\right)^{1 / \alpha}, \quad x=\left(x_{1}, \ldots, x_{n}\right)^{\prime} \in \mathbb{R}^{n} .
$$

In particular, our results lead to further analogues of Pólya's criterion for $\|\cdot\|_{\alpha^{-}}$ dependent functions, $\alpha>0$.

\section{Proof of the CRITERION}

The idea for the proof of Theorem 1.1 is straightforward. Proposition 2.1 shows that if $\alpha=1 / 2$, then the theorem gives necessary and sufficient conditions on $\varphi$ to admit a scale mixture representation in terms of a certain basis function $\varphi_{k+l, l} \in \Phi_{2 l+1}$. Specifically, we construct $\varphi_{k+l, l}$ as a compactly supported cut-off polynomial. For $l \geq 1$, define $\varphi_{l, l} \in \Phi_{2 l+1}$ to be the Euclid's hat function,

$$
\varphi_{l, l}(t)=c_{l} \int_{t}^{1}\left(1-v^{2}\right)_{+}^{l} d v
$$

which is proportional to the radial part of the self-convolution of an indicator function supported on the unit ball in $\mathbb{R}^{2 l+1}$ (see 7], Section 2). Here, $u_{+}$is $u$ or 0 according to whether $u>0$ or $u \leq 0$, and $c_{l}$ is a constant such that $\varphi_{l, l}(0)=1$. To introduce $\varphi_{k+l, l}$ for $k \geq 1$ and $l \geq 0$, consider the operator

$$
I \varphi(t)=\int_{t}^{\infty} u \varphi(u) d u / \int_{0}^{\infty} u \varphi(u) d u \quad(t \geq 0)
$$

and define

$$
\varphi_{k+l, l}=I^{k} \varphi_{k+l, k+l}
$$

by a $k$-fold application of the operator to the Euclid's hat function $\varphi_{k+l, k+l}$. From the results of Matheron (18], Chapter I) and Wu [33, $\varphi_{k+l, l}$ is an element of $\Phi_{2 l+1}$. Furthermore, $\varphi_{k+l, l}(t)$ is a polynomial of degree $2(k+l)+1$ for $t \leq 1$ and vanishes for $t \geq 1$, and the associated radial function is $2 k$ times differentiable at the origin. The construction is a special case of Matheron's claviers sphériques ([18], Section II.5), and our notation follows the more explicit presentation of $\mathrm{Wu}$ [33]. Theorem 4.9 of Wendland [30] establishes the equivalence of Matheron's and Wu's seemingly different approaches.

Proposition 2.1. If $\alpha=1 / 2$, Theorem 1.1 gives necessary and sufficient conditions for a function $\varphi:[0, \infty) \rightarrow \mathbb{R}$ to be of the form

$$
\varphi(t)=\int_{(0, \infty)} \varphi_{k+l, l}(r t) d F(r) \quad(t \geq 0),
$$

where $\varphi_{k+l, l} \in \Phi_{2 l+1}$ is Wu's function (7), and $F$ is a probability measure on $(0, \infty)$. 
Proof. Suppose first that $\varphi$ is of the form (8). Then clearly $\varphi$ is continuous with $\varphi(0)=1$ and $\lim _{t \rightarrow \infty} \varphi(t)=0$. Furthermore, the construction of $\varphi_{k+l, l}$ implies that

$$
\eta_{1}(t)=\int_{(0, \infty)} \varphi_{k+l, k+l}(r t) d G(r)
$$

for $t>0$, where $\varphi_{k+l, k+l}$ is the Euclid's hat function (5), and $G$ is nondecreasing with $G(0+)=0$ but not necessarily bounded. The interchange in the order of the differentiation and integration is justified by the compact support and boundedness of Wu's functions. Then if $\alpha=1 / 2$, the arguments on page 96 in [7] show that $\eta_{2}(t)$ is convex for $t>0$.

Conversely, assume that $\varphi$ satisfies the conditions of Theorem 1.1 with $\alpha=1 / 2$. Then the function $-\eta_{1}^{\prime}\left(t^{1 / 2}\right)$ satisfies the conditions of Lemma 4.3 in [7] with $m=$ $k+l+1$. (Assumption (b) of the lemma holds, because $\lim _{t \rightarrow \infty} \varphi(t)=0$.) Thus, $\eta_{1}^{\prime}$ admits a representation of the form

$$
\eta_{1}^{\prime}(t)=-\int_{(0, \infty)}\left(1-r t^{2}\right)_{+}^{k+l} d H(r)
$$

for $t>0$, where $H$ is nondecreasing with $H(0+)=0$. Integration with respect to $t$ shows that $\eta_{1}$ is of the form

$$
\eta_{1}(t)=\int_{(0, \infty)} \varphi_{k+l, k+l}(r t) d G(r)
$$

for $t>0$, where $\varphi_{k+l, k+l}$ is the Euclid's hat function, and $G$ is nondecreasing with $G(0+)=0$. Here and in the following, the integration constant is zero, because $\lim _{t \rightarrow \infty} \varphi(t)=0$; and the interchange in the order of the integration is permissible because the integrand is nonnegative. In view of Eqs. (3) and (7), we conclude from a $k$-fold application of the operator $I$ to both sides of the equality (9) that $\varphi$ is of the form (8) for some nondecreasing function $F$ with $F(0+)=0$. Finally, $F$ can be identified with a probability measure on $(0, \infty)$, because $\varphi(0)=1$ and $\lim _{t \rightarrow \infty} \varphi(t)=0$.

The proof of Theorem 1.1 is now straightforward. If $\alpha=1 / 2$ and $n=2 l+1$ the theorem holds by the proposition, because $\varphi_{k+l, l}$ is an element of $\Phi_{2 l+1}$, and the class is closed under scale mixtures. If $\varphi$ satisfies the conditions of the theorem for some $\alpha>1 / 2$ it does so for $\alpha=1 / 2$ too, by Proposition 4.5 of [7]. Finally, the assertion of the theorem holds for $n=1, \ldots, 2 l+1$, because it holds for $n=2 l+1$ and the classes $\Phi_{n}$ are nonincreasing in $n$. The proof is complete.

A natural question here is whether the scale mixture representations of Wu's functions yield the strongest possible criteria of the Pólya type. A partial answer is contained in the following proposition, which refers to a similar construction of Wendland 30. For nonnegative integers $k$ and $l$, consider Wendland's compactly supported function

$$
\psi_{k+l+1, k}(t)=I^{k}(1-t)_{+}^{k+l+1} \quad(t \geq 0),
$$

which belongs to the class $\Phi_{2 l+1}$, and whose even continuation to the real line is $2 k$ times differentiable at zero. Proposition 2.2 shows that Theorem 1.1 characterizes the scale mixtures of Wendland's functions if $\alpha=1$. In view of Proposition 4.5 in [7], the scale mixtures of Wendland's function $\psi_{k+l+1, k}$ form a subclass of the scale mixtures of Wu's function $\varphi_{k+l, l}$, and the resulting criterion is weaker. 
TABLE 1. Upper bounds for Kuttner's function $k_{1}(\lambda)$.

\begin{tabular}{|c|c|c|c|c|c|c|c|c|c|}
\hline$\lambda$ & $3 / 2$ & $5 / 3$ & 1.7908 & 1.8085 & 1.8976 & 1.9138 & 1.9345 & 1.9480 & 1.9550 \\
\hline upper bound & 2.0000 & 3.0000 & 4.0000 & 5.0000 & 6.0000 & 7.0000 & 8.0000 & 9.0000 & 10.0000 \\
\hline
\end{tabular}

Proposition 2.2. If $\alpha=1$, Theorem 1.1 gives necessary and sufficient conditions for a function $\varphi:[0, \infty) \rightarrow \mathbb{R}$ to be of the form

$$
\varphi(t)=\int_{(0, \infty)} \psi_{k+l+1, k}(r t) d F(r) \quad(t \geq 0)
$$

where $\psi_{k+l+1, k} \in \Phi_{2 l+1}$ is Wendland's function (10), and $F$ is a probability measure on $(0, \infty)$.

We omit the proof of Proposition 2.2, because it is entirely analogous to that of Proposition 2.1. Both results show, in particular, that Theorem 1.1 applies to radial functions with $2 k$ (or less) continuous derivatives at zero, for any degree of smoothness $k$. Examples abound, and applications in spatial statistics will be discussed elsewhere. Here, we focus on an application to a problem of Kuttner [14] and Golubov [10].

\section{The Kuttner-Golubov Problem}

In this section we consider the question for which values of $\lambda>0$ and $\kappa>0$ the truncated power function

$$
\varphi_{\lambda, \kappa}(t)=\left(1-t^{\lambda}\right)_{+}^{\kappa}
$$

is an element of the class $\Phi_{n}$. The problem can be formulated equivalently in terms of summability and positivity for Fourier series and Bessel integrals, and we refer to Kuttner [14], Golubov [10], Misiewicz and Richards [20, Berens and Xu [3], and Zastavnyi [35, 36] for these relations. The univariate case, $n=1$, has an interesting history and dates back to the works of Wintner [32] and Kuttner [14] (see [9] for a more detailed discussion). It is easily seen that $\varphi_{\lambda, \kappa}$ is not an element of $\Phi_{1}$ if $\lambda \geq 2$, regardless of the value of $\kappa$. Kuttner then showed that there exists a function $k_{1}(\lambda), \lambda \in(0,2)$, such that $\varphi_{\lambda, \kappa}$ is an element of $\Phi_{1}$ if and only if $\kappa \geq k_{1}(\lambda)$. The function $k_{1}(\lambda)$ is continuous and strictly increasing, $\lim _{\lambda \rightarrow 0} k_{1}(\lambda)>0, k_{1}(1)=1$, $\lim _{\lambda \rightarrow 2} k_{1}(\lambda)=\infty$, and $k_{1}(\lambda)>\lambda$ if $\lambda \neq 1$.

Let us now apply Theorem 1.1 for $\alpha=1 / 2$ and $l=0$ to find upper bounds on Kuttner's function $k_{1}(\lambda)$. These are summarized in Table 1. The estimates for $\lambda=$ $5 / 3, \lambda=1.7908$, and $\lambda=1.8085$ result from an application of the criterion for $k=1$ and have been discussed in the previous paper [7]. Generally, computer algebra systems allow for straightforward checks of the convexity of $\eta_{2}$ or the nonnegativity of $\eta_{2}^{\prime \prime}$, respectively. In fact, an application of Theorem 1.1 to the truncated power function (11) amounts to a check whether a polynomial is nonnegative on the unit interval. The calculations for the estimates in Table 1 have been performed with Maple [11. The fifth and sixth estimates are based on Theorem 1.1 with $k=2$, and for the last three estimates, the criterion with $k=3$ was used. The estimate for $\lambda=3 / 2$ is based on a different approach. In this specific case, the nonnegativity of the Bessel integral (2) can be proved directly.

Proposition 3.1. For Kuttner's function, $k_{1}(3 / 2) \leq 2$. 
Proof. We need to show that $\varphi(t)=\left(1-t^{3 / 2}\right)_{+}^{2}$ belongs to the class $\Phi_{1}$. From (22), this is true if and only if

$$
\begin{aligned}
\widehat{\varphi}(s) & =\int_{0}^{1}\left(1-t^{3 / 2}\right)^{2} \cos (t s) d t \\
& =\frac{3}{2} s^{-4}\left(\left(2 \pi s^{3}\right)^{1 / 2} C\left((2 s / \pi)^{1 / 2}\right)-4(\cos s+s \sin s-1)\right) \geq 0
\end{aligned}
$$

for $s>0$, where

$$
C(s)=\int_{0}^{s} \cos \left(\frac{\pi}{2} u^{2}\right) d u
$$

is a Fresnel integral. Substituting $\frac{\pi}{2} s^{2}$ for $s$, we get the equivalent condition that

$$
h_{0}(s)=\frac{\pi^{2}}{4} s^{3} C(s)-2 \cos \left(\frac{\pi}{2} s^{2}\right)-\pi s^{2} \sin \left(\frac{\pi}{2} s^{2}\right)+2 \geq 0
$$

for $s>0$. A straightforward albeit tedious calculation shows that $h_{0}(s)>0$ if $s \geq \sqrt{2}$. Let us now define

$$
h_{1}(s)=\frac{4}{3}(\pi s)^{-2} h_{0}^{\prime}(s)=C(s)-s \cos \left(\frac{\pi}{2} s^{2}\right)
$$

so that

$$
h_{1}^{\prime}(s)=\pi s^{2} \cos \left(\frac{\pi}{2} s^{2}\right) .
$$

Then $h_{1}(0)=0$ and $h_{1}^{\prime}(s) \geq 0$ for $s \in(0, \sqrt{2})$. Therefore, $h_{1}(s)$ and $h_{0}^{\prime}(s)$ are nonnegative for $s \in(0, \sqrt{2})$. Since $h_{0}(0)=0$, we conclude that $h_{0}(s) \geq 0$ if $s \in(0, \sqrt{2})$, and the proof is complete.

Let us now turn to the multivariate case. The question whether the truncated power function $\varphi_{\lambda, \kappa}$ belongs to the classes $\Phi_{n}, n=1,2, \ldots$, has been addressed by Golubov [10]. He showed that for all $n=1,2, \ldots$, there exists a function $k_{n}(\lambda)$, $\lambda \in(0,2)$, such that $\varphi_{\lambda, \kappa}$ is an element of $\Phi_{n}$ if and only if $\kappa \geq k_{n}(\lambda)$. The function $k_{n}(\lambda)$ is continuous and nondecreasing in $\lambda, \lim _{\lambda \rightarrow 0} k_{n}(\lambda)>0, \lim _{\lambda \rightarrow 2} k_{n}(\lambda)=\infty$, and $k_{n}(\lambda) \geq(n-1) / 2+\lambda$ with equality if $\lambda=1$. It is also clear that $k_{n}(\lambda)$ is nondecreasing in $n$. However, finite upper bounds on $k_{n}(\lambda)$ have not been available for $n>1$ and $\lambda>1$. Table 2 provides upper bounds for $n=3$ and $\lambda>1$. The first estimate, $k_{3}(3 / 2) \leq 3$, is given in Proposition 3.2. It is based on a direct proof of the nonnegativity of the Bessel integral (2). The second and third, the following three, and the last two estimates in the table, respectively, result from an application of Theorem 1.1 with $\alpha=1 / 2 ; l=1$; and $k=1, k=2$, and $k=3$; respectively. Again, the computer algebra system Maple has been used to check the nonnegativity of $\eta_{2}^{\prime \prime}$. Similar estimates for larger values of $n$ can be obtained from an application of the criterion for larger values of $l$.

Proposition 3.2. For the Kuttner-Golubov function, $k_{3}(3 / 2) \leq 3$.

Proof. We need to show that $\varphi(t)=\left(1-t^{3 / 2}\right)_{+}^{3}$ is an element of $\Phi_{3}$. From (2) , this is true if and only if

$$
\widehat{\varphi}(s)=\int_{0}^{1}\left(1-t^{3 / 2}\right)^{3} t \sin (t s) d t \geq 0
$$


TABLE 2. Upper bounds for the Kuttner-Golubov function $k_{3}(\lambda)$.

\begin{tabular}{|c|c|c|c|c|c|c|c|c|}
\hline$\lambda$ & $3 / 2$ & 1.6995 & 1.7630 & 1.8095 & 1.8802 & 1.8952 & 1.9235 & 1.9367 \\
\hline upper bound & 3.0000 & 4.0000 & 5.0000 & 6.0000 & 7.0000 & 8.0000 & 9.0000 & 10.0000 \\
\hline
\end{tabular}

for $s>0$. Arguments in analogy to those in the proof of Proposition 3.1 show that $\widehat{\varphi}(s)$ is nonnegative if and only if

$$
\begin{aligned}
h_{0}(s)= & 1155 S(s)+5 \pi^{3} s^{6} C(s)+128 \pi s^{3} \\
& +\left(13 \pi^{2} s^{4}-1155\right) s \sin \left(\frac{\pi}{2} s^{2}\right)+257 \pi s^{3} \cos \left(\frac{\pi}{2} s^{2}\right) \geq 0
\end{aligned}
$$

for $s>0$, where $S$ and $C$ are Fresnel integrals defined by

$$
S(s)=\int_{0}^{s} \sin \left(\frac{\pi}{2} u^{2}\right) d u
$$

and (12), respectively. Straightforward but tedious estimates show that $h_{0}(s)>0$ if $s \geq \sqrt{2}$. Then consider

$$
\begin{aligned}
h_{1}(s)= & \left(6 \pi s^{2}\right)^{-1} h_{0}^{\prime}(s)=64+5 \pi^{2} s^{3} C(s) \\
& \quad+\left(3 \pi^{2} s^{4}-64\right) \cos \left(\frac{\pi}{2} s^{2}\right)-32 \pi s^{2} \sin \left(\frac{\pi}{2} s^{2}\right), \\
h_{2}(s)= & \left(3 \pi^{2} s^{2}\right)^{-1} h_{1}^{\prime}(s)=5 C(s)-5 s \cos \left(\frac{\pi}{2} s^{2}\right)-\pi s^{3} \sin \left(\frac{\pi}{2} s^{2}\right), \\
h_{3}(s)= & \left(\pi s^{2}\right)^{-1} h_{2}^{\prime}(s)=2 \sin \left(\frac{\pi}{2} s^{2}\right)-\pi s^{2} \cos \left(\frac{\pi}{2} s^{2}\right), \\
h_{4}(s)= & h_{3}^{\prime}(s)=\pi^{2} s^{3} \sin \left(\frac{\pi}{2} s^{2}\right) .
\end{aligned}
$$

Note that $h_{i}(0)=0$ for $i=0,1,2,3$, and $h_{4}(s)=h_{3}^{\prime}(s) \geq 0$ for $s \in(0, \sqrt{2})$. Proceeding inductively, we see that $h_{i}(s) \geq 0$ for $i=3,2,1,0$ and $s \in(0, \sqrt{2})$. The proof is complete.

\section{4. $\|\cdot\|_{\alpha}$-DEPEndent Positive Definite FunCtions}

This final section is concerned with positive definite functions $f(x)=\varphi\left(\|x\|_{\alpha}\right)$, $x \in \mathbb{R}^{n}$, which depend on the $l_{\alpha}$-(quasi-)norm $\|x\|_{\alpha}=\left(\left|x_{1}\right|^{\alpha}+\cdots+\left|x_{n}\right|^{\alpha}\right)^{1 / \alpha}$, $\alpha>0$, or on the maximum norm, $\|x\|_{\infty}=\max \left(\left|x_{1}\right|, \ldots,\left|x_{n}\right|\right)$. We denote by

$$
\Phi_{n}(\alpha), \quad n=1,2, \ldots, \quad \alpha>0,
$$

the class of the continuous functions $\varphi:[0, \infty) \rightarrow \mathbb{R}$ which are such that $\varphi(0)=1$ and $f(x)=\varphi\left(\|x\|_{\alpha}\right), x \in \mathbb{R}^{n}$, is positive definite. Thus, $\Phi_{1}(\alpha)=\Phi_{1}$, independently of $\alpha$, and $\Phi_{n}(2)$ is the class $\Phi_{n}$ defined in Section 1, For recent reviews of $\|\cdot\|_{\alpha^{-}}$ dependent positive definite functions and connections to the isometric theory of Banach spaces, we refer to Koldobsky [13, Misiewicz [19], and Gneiting [5].

Zastavnyi 35, 36 investigated for which values of $\lambda>0$ and $\kappa>0$ the truncated power function $\varphi_{\lambda, \kappa}(t)=\left(1-t^{\lambda}\right)_{+}^{\kappa}$ is an element of the class $\Phi_{n}(\alpha)$. He showed that for all $n \geq 1$ and $\alpha>0$ there exists a nondecreasing but not necessarily finite function $k_{n, \alpha}(\lambda), \lambda \in(0,2)$, such that $\varphi_{\lambda, \kappa}$ is an element of $\Phi_{n}(\alpha)$ if and only if $\kappa \geq k_{n, \alpha}(\lambda)$. Clearly $k_{1, \alpha}(\lambda)$ is Kuttner's function $k_{1}(\lambda)$, independently of $\alpha$, and $k_{n, 2}(\lambda)$ is the Kuttner-Golubov function $k_{n}(\lambda)$. If we fix $\lambda=1$, then $k_{n, \alpha}(1)$ is the Richards-Askey function which the author introduced in [5]. Zastavnyi [35] established an interesting connection to Schoenberg's [28] classical question whether 
$\varphi(t)=\exp \left(-t^{\lambda}\right)$ is an element of $\Phi_{n}(\alpha)$. Specifically, he showed that if $\lambda \in(0,2)$, then $k_{n, \alpha}(\lambda)$ is finite if and only if $\varphi(t)=\exp \left(-t^{\lambda}\right)$ belongs to the class $\Phi_{n}(\alpha)$. Thus, Koldobskiî's [12 and Zastavnyi's 34] solution to the Schoenberg problem implies that $k_{n, \alpha}(\lambda)$ is finite if (i) $n=1, \alpha \in(0, \infty], \lambda \in(0,2)$; (ii) $n \geq 2$, $\alpha \in(0,2), \lambda \in(0, \alpha]$; (iii) $n \geq 2, \alpha=2, \lambda \in(0,2)$; and (iv) $n=2, \alpha \in(2, \infty]$, $\lambda \in(0,1]$; and infinite otherwise. Numerical values of $k_{n, \alpha}(\lambda)$ are known in special cases only: $k_{n, 2}(1)=(n+1) / 2\left(\right.$ Golubov [10]); $k_{2,2}(1 / 2)=1$ (Pasenchenko [23]); $k_{n, 1}(1)=2 n-1$ (Berens and $\mathrm{Xu}[3]$ ); and $k_{2, \infty}(1)=3$. Furthermore, various estimates are known. Golubov [10] showed that $k_{n, 2}(\lambda) \geq(n-1) / 2+\lambda$, and Zastavnyi's [35] Corollary 3 implies that $k_{2, \alpha}(1) \leq 3$ if and only if $\alpha \geq 1$. The following result shows that $k_{n, \alpha}(\lambda)$ grows at least logarithmically, and at most linearly, with $n$. The crucial upper estimate is due to Zastavnyi [35].

Theorem 4.1. If $0<\lambda \leq \alpha<2$, then

$$
-\frac{\ln (2 n)}{\ln \left(1-2^{-\lambda / \max (\alpha, 1)}\right)} \leq k_{n, \alpha}(\lambda) \leq k_{n, \alpha}(\alpha) \leq n\left(k_{1}(\alpha)+1\right)-1 .
$$

Proof. To prove the lower bound, apply Lemma 3.7 of [5] to the function $\varphi(t)=$ $\left(1-t^{\lambda}\right)_{+}^{\kappa}$. The second inequality is immediate because $k_{n, \alpha}(\lambda)$ is nondecreasing in $\lambda$, and the upper estimate is given in Corollary 4 of Zastavnyi 35 .

In view of the upper inequality in (13), the bounds on Kuttner's function in Table 1 provide finite upper estimates for the general function $k_{n, \alpha}(\lambda)$, for all $n$, and for a very wide range of $\alpha$ and $\lambda$. Clearly, upper estimates for larger values of $\alpha$ will result from an application of Theorem 1.1 for $l=0$ and larger values of $k$. It is also interesting to observe that the upper estimate in (13) is attained if $\alpha=1$. After acceptance of this paper, Professor Victor P. Zastavnyi kindly pointed to the relevance of the results in Liflyand et al. [17]. Details and an associated lower bound on $k_{n, \alpha}(\alpha)$ will be discussed elsewhere.

The most attractive feature of the problem is certainly the close connection to analogues of Pólya's criterion. Specifically, if $\varphi_{\lambda, \kappa}(t)=\left(1-t^{\lambda}\right)_{+}^{\kappa}$ is an element of the class $\Phi_{n}(\alpha)$, then so are its scale mixtures of the form

$$
\varphi(t)=\int_{(0, \infty)}\left(1-r t^{\lambda}\right)_{+}^{\kappa} d F(r) \quad(t \geq 0)
$$

where $F$ is a probability measure on $(0, \infty)$. For $\kappa$ a nonnegative integer Proposition 3.5 of Gneiting [5] (see also Williamson 31] and Lévy [16]) characterizes the scale mixtures concisely. A continuous function $\varphi:[0, \infty) \rightarrow \mathbb{R}$ with $\varphi(0)=1$ and $\lim _{t \rightarrow \infty} \varphi(t)=0$ is of the form (14) if and only if

$$
\left(-\frac{d}{d t}\right)^{\kappa-1} \varphi\left(t^{1 / \lambda}\right)
$$

is convex for $t>0$. Thus, any finite upper bound on $k_{n, \alpha}(\lambda)$ will immediately provide a criterion of the Pólya type.

Let us assume in the following that $\varphi:[0, \infty) \rightarrow \mathbb{R}$ is a continuous function with $\varphi(0)=1$ and $\lim _{t \rightarrow \infty} \varphi(t)=0$. Since $k_{2 n-1,2}(1)=n$, we conclude that $\varphi \in \Phi_{2 n-1}(2)$ if $(-1)^{n-1} \varphi^{(n-1)}(t)$ is convex for $t>0$. This is the beautiful Askey's criterion [1], which reduces to Pólya's theorem if $n=1$. Richards [25] called for $\alpha$-analogues of Askey's criterion and, indeed, these have been found. Since $k_{n, 1}(1)=2 n-1$, we see that $\varphi \in \Phi_{n}(1)$ if $\varphi^{(2 n-2)}(t)$ is convex for $t>0$. This 
result again reduces to Pólya's theorem if $n=1$. It was established independently by Berens and Xu [3] and Gneiting [5]. Finally, the criteria of Zastavnyi [35, 36] and Gneiting [8] stem from the facts that $k_{2, \alpha}(1) \leq 3$ if $\alpha \in[1, \infty]$, and $k_{2,2}(1 / 2)=1$, respectively. Clearly, our findings in Section 3 provide very many new results of this type. For example, we conclude from Proposition 3.1 that $\varphi$ belongs to the class $\Phi_{1}$ if $-t^{-1 / 3} \varphi^{\prime}\left(t^{2 / 3}\right)$ is convex for $t>0$. In particular, the univariate function $f(x)=\varphi(|x|)$ is positive definite if

$$
3 t \varphi^{\prime \prime}(t)-2 t^{2} \varphi^{\prime \prime \prime}(t)-2 \varphi^{\prime}(t) \geq 0
$$

for $t>0$. Similarly, Proposition 3.2, our estimates in the tables, and Theorem 4.1 lead to further analogues of Pólya's criterion, which we leave to the reader.

As our final remark, a criterion of this type allows for functions $f(x)=\varphi\left(\|x\|_{\alpha}\right)$ which are differentiable of order at most one at zero. A natural question is whether there are $\alpha$-analogues of Pólya's theorem that apply to functions which are smoother at the origin. If $\alpha=2$, Theorem 1.1 provides this kind of criterion. If $\alpha \neq 2$, the answer is in the negative. Proposition 4.1.3 of Misiewicz and Scheffer [21] and the remarks thereafter imply that an $\|\cdot\|_{\alpha}$-dependent positive definite function on $\mathbb{R}^{n}$ has at most one continuous derivative at the origin, unless $n=1$ or $\alpha=2$.

\section{ACKNOWLEDGEMENTS}

I am grateful to Martin Schlather for his suggestion to generalize the criterion in [9, which led me to the formulation of Theorem 1.1

\section{REFERENCES}

1. Askey, R., Radial Characteristic Functions, University of Wisconsin-Madison, Mathematics Research Center, 1262, 1973.

2. Askey, R., Some characteristic functions of unimodal distributions, J. Math. Anal. Appl., 50 (1975), 465-469. MR 51:6910

3. Berens, H. and Xu, Y., l-1 summability of multiple Fourier integrals and positivity, Math. Proc. Cambridge Philos. Soc., 122 (1997), 149-172. MR 98g:42035

4. Bochner, S. and Chandrasekharan, K., Fourier Transforms, Princeton University Press, 1949. MR 11:173d

5. Gneiting, T., On $\alpha$-symmetric multivariate characteristic functions, J. Multivariate Anal., 64 (1998), 131-147. MR 99h:60025

6. Gneiting, T., On the derivatives of radial positive definite functions, J. Math. Anal. Appl., 236 (1999), 86-93. MR 2000k:42012

7. Gneiting, T., Radial positive definite functions generated by Euclid's hat, J. Multivariate Anal., 69 (1999), 88-119. MR 2000g:60022

8. Gneiting, T., A Pólya type criterion for radial characteristic functions in $\mathbb{R}^{2}$, Exposition. Math., 17 (1999), 181-183. MR 2000e:60024

9. Gneiting, T., Kuttner's problem and a Pólya type criterion for characteristic functions, Proc. Amer. Math. Soc., 128 (2000), 1721-1728. MR 2000j:42016

10. Golubov, B. I., On Abel-Poisson type and Riesz means, Anal. Math., 7 (1981), 161-184. MR 83b:42015

11. Heal, K. M. and Hansen, M. L. and Rickard, K. M., Maple V Learning Guide, Springer, New York, 1996.

12. Koldobskiī, A. L., Schoenberg's problem on positive definite functions, St. Petersburg Math. J., 3 (1992), 563-570. MR 93c:42014

13. Koldobsky, A., Positive definite functions, stable measures, and isometries on Banach spaces, Interaction Between Functional Analysis, Harmonic Analysis, and Probability, Lecture Notes in Pure and Appl. Math., 175, Marcel Dekker, 1996, 275-290. MR 96k:46016

14. Kuttner, B., On the Riesz means of a Fourier series (II), J. London Math. Soc., 19 (1944), 77-84. MR 7:59d 
15. Letac, G. and Rahman, Q. I., A factorisation of the Askey's characteristic function (1 $\left.\|t\|_{2 n+1}\right)_{+}^{n+1}$, Ann. Inst. H. Poincaré Probab. Statist., 22 (1986), 169-174. MR 87i:42027

16. Lévy, P. , Extensions d'un théorème de D. Dugué et M. Girault, Z. Wahrscheinlichkeitstheorie verw. Gebiete, 1 (1962), 159-173. MR 26:3096

17. Liflyand, E. R., Ramm, A. G. and Zaslavsky, A. I., Estimates from below for Lebesgue constants, J. Fourier Anal. Appl., 2 (1996), 287-301. MR 97g:42007

18. Matheron, G., Les variables régionalisées et leur estimation, Masson, Paris, 1965.

19. Misiewicz, J. K., Sub-stable and pseudo-isotropic processes, connections with the geometry of sub-spaces of $L_{\alpha}$-spaces, Dissertationes Math., 358 (1996), 1-91. MR 97k:60040b

20. Misiewicz, J. K. and Richards, D. St. P., Positivity of integrals of Bessel functions, SIAM J. Math. Anal., 25 (1994), 596-601. MR 95i:33004

21. Misiewicz, J. K. and Scheffer, C. L., Pseudo isotropic measures, Nieuw Arch. Wisk. IV, 8 (1990), 111-152. MR 92e:60007

22. Mittal, Y., A class of isotropic covariance functions, Pacific J. Math., 64 (1976), 517-538. MR 54:14068

23. Pasenchenko, O. Yu., Sufficient conditions for the characteristic function of a two-dimensional isotropic distribution, Theory Probab. Math. Statist., 53 (1996), 149-152. MR 98b:60033

24. Pólya, G., Remarks on Characteristic Functions, Proceedings of the Berkeley Symposium on Mathematical Statistics and Probability, University of California Press, 1949, 115-123. MR 10:463c

25. Richards, D. St. P., Positive definite symmetric functions on finite dimensional spaces. I. Applications of the Radon transform, J. Multivariate Anal., 19 (1986), 280-298. MR 88c:60043a

26. Sasvári, Z., On a classical theorem in the theory of Fourier integrals, Proc. Amer. Math. Soc., 126 (1998), 711-713. MR 98i:60013

27. Schoenberg, I. J., Metric spaces and completely monotone functions, Ann. of Math., 39 (1938), 811-841.

28. Schoenberg, I. J., Metric spaces and positive definite functions, Trans. Amer. Math. Soc., 44 (1938), 522-536. CMP 95:18

29. Trigub, R. M., A criterion for a characteristic function and a Polyá type criterion for radial functions of several variables, Theory Probab. Appl., 34 (1989), 738-742. MR 91f:60033

30. Wendland, H., Piecewise polynomial, positive definite and compactly supported radial functions of minimal degree, Adv. Comput. Math., 4 (1995), 389-396. MR 96h:41025

31. Williamson, R. E., Multiply monotone functions and their Laplace transforms, Duke Math. J., 23 (1956), 189-207. MR 17:1061d

32. Wintner, A., On a family of Fourier transforms, Bull. Amer. Math. Soc., 48 (1942), 304-308. MR 3:232a

33. Wu, Z., Compactly supported positive definite radial functions, Adv. Comput. Math., 4 (1995), 283-292. MR 97g:65031

34. Zastavnyi, V. P., Positive-definite functions that depend on a norm, Russian Acad. Sci. Dokl. Math., 46 (1993), 112-114. MR 94k:42026

35. Zastavnyi, V. P., On positive definiteness of some functions, Dokl. Math., 365 (1999), 159161. MR 2000i: 42005

36. Zastavnyi, V. P., On positive definiteness of some functions, J. Multivariate Anal., 73 (2000), 55-81. CMP 2000:14

Department of Statistics, Box 354322, University of Washington, Seattle, WashingTON 98195

E-mail address: tilmann@stat.washington.edu 